\title{
A gravitational-wave probe of effective quantum gravity
}

\author{
Stephon Alexander* Lee Samuel Finn $\oplus^{\dagger}$ and Nicolás Yunes ${ }^{\dagger}$ \\ The Pennsylvania State University, University Park, PA 16802, USA
}

(Dated: October 25, 2018)

\begin{abstract}
The Green-Schwarz anomaly-cancelling mechanism in string theories requires a Chern-Simons term in the Einstein-Hilbert action, which leads to an amplitude birefringence of spacetime for the propagation of gravitational waves. While the degree of birefringence may be intrinsically small, its effects on a gravitational wave will accumulate as the wave propagates. The proposed Laser Interferometer Space Antenna (LISA) will be sensitive enough to observe the gravitational waves from sources at cosmological distances great enough that interesting bounds on the Chern-Simons may be found. Here we evaluate the effect of a Chern-Simons induced spacetime birefringence to the propagation of gravitational waves from such systems. We find that gravitational waves from in coalescing binary black hole system are imprinted with a signature of Chern-Simons gravity. This signature appears as a time-dependent change in the apparent orientation of the binary's orbital angular momentum with respect to the observer line-of-sight, with the change magnitude reflecting the integrated history of the Chern-Simons coupling over the worldline of a radiation wavefront. While spin-orbit coupling in the binary system will also lead to an evolution of the system's orbital angular momentum, the time dependence and other details of this real effect are different than the apparent effect produced by Chern-Simons birefringence, allowing the two effects to be separately indentified. In this way gravitational wave observations with LISA may thus provide our first and only opportunity to probe the quantum structure of spacetime over cosmological distances.
\end{abstract}

PACS numbers: 11.25.Wx, 95.55.Ym, 04.60.-m, 04.80.Cc

\section{INTRODUCTION}

"Gravitational wave" is the name we give to a short-wavelength feature of the structure of spacetime, the arena within which all other phenomena play-out their roles. As such, the direct observation of gravitational waves offers an unprecedented opportunity to explore the environment that both enables and constrains the action of the broader phenomena of nature. Here we describe an observational probe of quantum gravity made possible by the forthcoming generation of space-based gravitational wave detectors.

In most corners of the perturbative string theory moduli space (i.e. Type IIB, I, Heterotic) in four-dimensional compactifications, the addition of a parity-violating Chern-Simons term to the Einstein-Hilbert is required for mathematical consistency [58]. Furthermore in the presence of the Ramond-Ramond scalar (D-instanton charge), the Chern-Simons term is induced in all string theories due to duality symmetries [1, 2]. The Chern-Simons term is not unique to string theory, but in fact it could also arise in Loop Quantum Gravity, where the coupling is not necessarily limited to small values. In the strong gravity sector of this framework, this term arises to ensure invariance under large gauge transformations of the Ashtekar connection variables [3]. This correction plays the same role in string theory that an analogous anomaly-canceling correction to the quantum-chromodynamics path integral plays in the Standard Model [4]. While the anomaly-cancelling field in the Standard Model case interacts with photons, leading to significant observational constraints on its coupling, the anomaly canceling term considered here only affects the gravitational sector of the theory and is mostly unconstrained by observation [5, 6, 7].

The Chern-Simons correction to the Einstein-Hilbert action, Chern-Simons gravity [8], has been recently studied in connection with tests both in the cosmological [2, 9, 10, 11] and the weak-gravitational contexts [5, 6, 7]. In the context of gravitational wave theory, Chern-Simons gravity leads to an amplitude birefringence of spacetime for gravitational wave propagation 2, 8, 12]: i.e., a polarization dependent amplification/attenuation of wave amplitude with distance propagated. Observation of gravitational waves that have propagated over cosmological distances, such as will be possible with the Laser Interferometer Gravitational Wave Observatory (LISA) 13, 14, 15], provide the opportunity to measure or bound the magnitude of the birefringence and, correspondingly, provide the first experimental constraints

\footnotetext{
*Center for Fundamental Theory, Institute for Gravitational Physics and Geometry, Department of Physics

${ }^{\dagger}$ Center for Gravitational Wave Physics, Institute for Gravitational Physics and Geometry, Department of Physics, Department of Astronomy and Astrophysics

${ }^{\ddagger}$ Center for Gravitational Wave Physics, Institute for Gravitational Physics and Geometry, Department of Physics
} 
on string theory models of gravity.

Gravitational wave observations have long been recognized as a tool for testing our understanding of gravity (see 16] for a recent review). Eardley and collaborators 17, 18] first proposed a far-field test of all metric theories of gravity through gravitational wave observations. Finn [19], and later Cutler and Lindblom [20], proposed a means of realizing these measurements using a space-based detector in a circumsolar orbit observing solar oscillations in the far-zone field. Ryan [21] argued that observations of the phase evolution of the gravitational waves emitted during the gravitationalwave driven inspiral of, e.g., a neutron star or stellar mass black hole into a supermassive black hole could be used to "map-out" the spacetime metric in the vicinity of the black hole horizon, testing the predictions of general relativity in the regime of strong-fields. There have been several proposals describing different ways in which gravitational wave observations could be used to place bounds on the graviton Compton-wavelength [22, 23, 24, 25, 26, 27], the existence of a scalar component to the gravitational interaction [25, 26, 27, 28], and the existence of other corrections to general relativity as manifest in some fundamental, dimensionful length scale [29, 30]. The measurements we propose here are, we believe, the first example of a direct model-independent probe of string theory and quantum gravity with gravitational waves.

In section \ we review Chern-Simons modified gravity, focusing attention on the scale of the Chern-Simons term and its effect on the propagation of gravitational waves in a cosmological background. In section III we evaluate the observational consequences of the Chern-Simons term in the context of ground- and space-based gravitational wave detectors. In section $\mathbf{V}$ we summarize our conclusions and discuss avenues of future research.

Conventions used in relativity work and conventions used in quantum field theory work are often at odds. We follow the relativity conventions Misner, Thorne and Wheeler [31] in this work: in particular,

- Our metric has signature -+++ ;

- We label indices on spacetime tensors with greek characters and use latin indices to label indices on tensors defined on spacelike slices;

- We use a semicolon in an index list to denote a covariant derivative (i.e., $\nabla_{\mathbf{V}} \mathbf{U}$ becomes $V^{\nu} U_{\mu ; \nu}$ ) and a comma to denote ordinary partial derivatives;

- Except where explicitly noted we work in geometric units, wherein $G=c=1$ for Newtonian gravitational constant $G$ speed of light $c$.

Note that in geometric units, units of mass and length are interchangeable (i.e., $G / c^{2}$ has units of (length) $/($ mass)). This is in contrast to Planck units $(\hbar=c=1)$, where units of mass and units of inverse length are interchangeable (i.e., $\hbar / c$ has units of $($ mass $) \times($ length $))$.

\section{CHERN-SIMONS MODIFIED GRAVITY}

\section{A. Brief Review}

In this subsection we review the modification to classical General Relativity by the inclusion of a Chern-Simons term, based on [8, 12]. All four dimensional compactifications of string theory lead, via the Green-Schwarz anomaly canceling mechanism, to the presence of a four dimensional gravitational Chern-Simons term [1]. Chern-Simons forms are formally defined for odd dimensions, with the 3-form of particular interest for gauge theories. By introducing an embedding coordinate, which may be dynamical, Jackiw and Yi [8] described a Chern-Simons correction to the Einstein-Hilbert action:

$$
S_{\mathrm{CS}}=\frac{1}{64 \pi} \int d^{4} x \theta R^{*} R
$$

where $\theta$ is (a functional of) the embedding coordinate,

$$
R^{*} R=\frac{1}{2} R_{\alpha \beta \gamma \delta} \epsilon^{\alpha \beta \mu \nu} R^{\gamma \delta}{ }_{\mu \nu}
$$

and $\epsilon^{\alpha \beta \gamma \delta}$ is the Levi-Civita tensor density. The variation with respect to the metric of this contribution to the total action (which includes the Einstein-Hilbert action plus the action corresponding to any additional matter fields) yields [8]

$$
\delta S_{\mathrm{CS}}=-\frac{1}{16 \pi} \int d^{4} x \sqrt{-g} C_{\alpha \beta} \delta g^{\alpha \beta}
$$


where $g$ is the determinant of the metric and $C^{a b}$ is the C-tensor [59],

$$
C^{\alpha \beta}=-\frac{1}{\sqrt{-g}}\left[\theta_{, \epsilon} \epsilon^{\epsilon \gamma \delta(\alpha} \nabla_{\gamma} R^{\beta)} \delta-\nabla_{\delta} \theta_{, \gamma} \epsilon^{\mu \nu \gamma(\alpha} R^{\beta) \delta}{ }_{\mu \nu}\right],
$$

and the parenthesis in the superscript stand for symmetrization. The variation of $S_{\mathrm{CS}}$, the usual Einstein-Hilbert action, and the action of other matter fields leads to the equations of motion of Chern-Simons modified gravity:

$$
G_{\alpha \beta}+C_{\alpha \beta}=8 \pi T_{\alpha \beta}
$$

where $G_{a b}$ is the Einstein tensor (i.e., the trace-reversed Ricci tensor) and $T_{a b}$ is the stress-energy tensor of the matter fields.

By construction the divergence of the Einstein tensor $G_{\alpha \beta}$ vanishes. If $\theta$ is treated as a fixed, external quantity then general covariance, which requires $\nabla \cdot \mathbf{T}=0$, leads to the constraint $\nabla \cdot \mathbf{C}=0$, which is shown in [8] to be equivalent to $R^{*} R=0$. Alternatively, if $\theta$ is a dynamical field, then variation of the action with respect to $\theta$ will lead to the same constraint on $R^{*} R$. Here we are interested in the propagation of gravitational waves in vacuum, where $\mathbf{T}=0$ and the constraint $\nabla \cdot \mathbf{C}=0$ is satisfied regardless of whether we view $\theta$ as a dynamical field or a fixed, externally-specified quantity.

\section{B. Linearized Chern-Simons gravitational waves}

Focus attention on gravitational wave perturbations to a Friedmann-Robertson-Walker (FRW) cosmological background in Chern-Simons gravity. Following [12], we can write the perturbed FRW line element as

$$
d s^{2}=a^{2}(\eta)\left[-d \eta^{2}+\left(\delta_{i j}+h_{i j}\right) d \chi^{i} d \chi^{j}\right]
$$

where $\eta$ is conformal time, $\chi^{i}$ are comoving spatial coordinates, $\delta_{i j}$ is the Euclidean metric, and $h_{i j}$ is the metric perturbation, which — for gravitational wave solutions — we can take to be transverse and traceless [60]. Introducing this perturbation into the field equations [Eq. (2.5)] leads to

$$
\square_{g} h^{j}{ }_{i}=-\frac{1}{a^{2}} \epsilon^{p j k}\left[\left(\theta^{\prime \prime}-2 \mathcal{H} \theta^{\prime}\right) \partial_{p} h_{k i}^{\prime}+\theta^{\prime} \partial_{p} \square_{g} h_{k i}\right]
$$

where we have introduced the notation

$$
\begin{aligned}
\prime & =\partial_{\eta} \\
\square_{g} & =\partial_{\eta}^{2}-\partial_{i} \partial_{j} \gamma^{i j}+2 \mathcal{H} \partial_{\eta} \\
\mathcal{H} & =a^{\prime} / a .
\end{aligned}
$$

Conformal time $\eta$ is related to proper time measured by an observer at rest with respect to the cosmological fluid via

$$
d t=a(\eta) d \eta
$$

correspondingly, the conformal Hubble function $\mathcal{H}$ is related to the Hubble function $H$ measured by an observer at rest with respect to slices of homogeneity via

$$
H \equiv \frac{\dot{a}}{a}=\frac{1}{a} \mathcal{H}
$$

where we have use dots to stand for derivatives with respect to cosmic time $t$.

Focus attention on plane-wave solutions to the wave equation [Eq. (2.7)]. With the ansatz

$$
h_{l m}\left(\eta, \chi^{l}\right)=\frac{\mathcal{A}_{l m}}{a(\eta)} e^{-i\left[\phi(\eta)-\kappa n_{k} \chi^{k}\right]},
$$

where the amplitude $\mathcal{A}_{l m}$, the unit vector in the direction of wave propagation $n_{k}$ and the conformal wavenumber $\kappa>0$ are all constant, we find that $\phi, \kappa$ and $\mathcal{A}_{i j}$ must satisfy

$$
\mathcal{D} \mathcal{A}_{i j}=-a^{-2} \epsilon^{p j k} n_{p} \mathcal{A}_{k i}\left[\left(\theta^{\prime \prime}-2 \mathcal{H} \theta^{\prime}\right)\left(\phi^{\prime}-i \mathcal{H}\right) \kappa+i \theta^{\prime} \kappa \mathcal{D}\right]
$$


where

$$
\mathcal{D}=-i \phi^{\prime \prime}-\left(\phi^{\prime}\right)^{2}-\mathcal{H}^{\prime}-\mathcal{H}^{2}+\kappa^{2}
$$

Since the Chern-Simons correction breaks parity, it is convenient to resolve $\mathcal{A}_{i j}$ into definite parity states, corresponding to radiation amplitude in the right- and left-handed polarizations $e_{i j}^{R}$ and $e_{i j}^{L}$ :

$$
\mathcal{A}_{i j}=\mathcal{A}_{R} e_{i j}^{R}+\mathcal{A}_{L} e_{i j}^{L}
$$

where

$$
\begin{aligned}
& e_{k l}^{R}=\frac{1}{\sqrt{2}}\left(e_{k l}^{+}+i e_{k l}^{\times}\right) \\
& e_{k l}^{L}=\frac{1}{\sqrt{2}}\left(e_{k l}^{+}-i e_{k l}^{\times}\right)
\end{aligned}
$$

and $e_{k l}^{+, \times}$are the usual linear polarization tensors [31]. It is straightforward to show that

$$
n_{i} \epsilon^{i j k} e_{k l}^{\mathrm{R}, \mathrm{L}}=i \lambda_{\mathrm{R}, \mathrm{L}}\left(e^{j}\right)^{\mathrm{R}, \mathrm{L}}
$$

where

$$
\begin{aligned}
& \lambda_{\mathrm{R}}=+1 \\
& \lambda_{\mathrm{L}}=-1 .
\end{aligned}
$$

With this substitution equation (2.14) becomes two decoupled equations, one for right-hand polarized waves and one for left-hand polarized waves:

$$
i \phi_{\mathrm{R}, \mathrm{L}}^{\prime \prime}+\left(\phi_{\mathrm{R}, \mathrm{L}}^{\prime}\right)^{2}+\mathcal{H}^{\prime}+\mathcal{H}^{2}-\kappa^{2}=\frac{i \lambda_{\mathrm{R}, \mathrm{L}}\left(\theta^{\prime \prime}-2 \mathcal{H} \theta^{\prime}\right)\left(\phi^{\prime}-i \mathcal{H}\right) \kappa / a^{2}}{\left(1-\lambda_{\mathrm{R}, \mathrm{L}} \kappa \theta^{\prime} / a^{2}\right)}
$$

The terms on the right-hand side of equation (2.18) are the Chern-Simons corrections to gravitational plane-wave propagation in a FRW spacetime. To understand the relative scale of these terms, we rewrite the equation in terms of the ratio $\phi^{\prime} / \kappa$ :

$$
\frac{y^{\prime}}{\kappa}+i\left(1-\gamma^{2} \Gamma^{2}-\delta \Delta-y^{2}\right)=\frac{\lambda_{\mathrm{R}, \mathrm{L}}(\epsilon E-2 \gamma \zeta \Gamma Z)}{1-\lambda_{\mathrm{R}, \mathrm{L}} \zeta Z}(y-i \gamma \Gamma)
$$

where

$$
\begin{array}{rlrlrl}
\gamma & y=\frac{\phi^{\prime}}{\kappa} & & \\
\kappa & & \text { and } & \Gamma & =\frac{\mathcal{H}}{\mathcal{H}_{0}} \\
\delta & =\frac{\mathcal{H}_{0}^{\prime}}{\kappa^{2}} & \text { and } & \Delta & =\frac{\mathcal{H}^{\prime}}{\mathcal{H}_{0}^{\prime}} \\
\epsilon & =\frac{\theta_{0}^{\prime \prime}}{a_{0}^{2}} & \text { and } & E & =\frac{\theta^{\prime \prime}}{a^{2} \epsilon} \\
\zeta & =\frac{\kappa \theta_{0}^{\prime}}{a_{0}^{2}} & & \text { and } & Z & =\frac{\kappa \theta^{\prime}}{a^{2} \zeta}
\end{array}
$$

and a subscript 0 indicates the present-day value of the functions $\theta^{\prime}, \theta^{\prime \prime}, \mathcal{H}, \mathcal{H}^{\prime}$ and $a$.

If we assume that $\theta$ and $\mathcal{H}$ evolve on cosmological timescales (i.e., $f^{\prime} \sim \mathcal{H} f$ ) then

$$
\epsilon^{2} \sim(\gamma \zeta)^{2} \ll \gamma^{2} \sim|\delta|
$$

Treating the terms in $\epsilon$ and $\gamma \zeta$ as perturbations, write the solution to equation (2.19a) as

$$
y=y_{0}+\epsilon y_{0,1}+\gamma \zeta y_{1,0}+\ldots
$$


where $y_{0}=\phi_{0}^{\prime} / \kappa$ is the solution to the unperturbed equation [i.e., the dispersion relation in an FRW cosmology, given by equation (2.19a ) with vanishing right-hand side]. The first corrections $y_{0,1}$ and $y_{1,0}$ owing to the Chern-Simons terms satisfy

$$
\begin{aligned}
& y_{0,1}^{\prime}-2 i \kappa y_{0} y_{0,1}=\lambda_{\mathrm{R}, \mathrm{L}} \kappa E y_{0} \\
& y_{1,0}^{\prime}-2 i \kappa y_{0} y_{1,0}=-2 \lambda_{\mathrm{R}, \mathrm{L}} \kappa \Gamma Z y_{0} .
\end{aligned}
$$

Requiring that the perturbation vanish at some initial (conformal) time $\eta_{i}$ the perturbations $y_{0,1}$ and $y_{1,0}$ satisfy

$$
\begin{aligned}
& y_{0,1}(\eta)=\lambda_{\mathrm{R}, \mathrm{L}} \mathcal{Y}[E](\eta) \\
& y_{1,0}(\eta)=-2 \lambda_{\mathrm{R}, \mathrm{L}} \mathcal{Y}[\Gamma Z](\eta)
\end{aligned}
$$

where

$$
\mathcal{Y}[g](\eta)=\kappa e^{2 i \phi_{0}(\eta)} \int_{\eta_{i}}^{\eta} d x e^{-2 i \phi_{0}(x)} y_{0}(x) g(x)
$$

Finally, the Chern-Simons correction to the accumulated phase as the plane-wave propagates from $\eta_{i}$ to $\eta$ is

$$
\delta \phi_{\mathrm{R}, \mathrm{L}}=\kappa \lambda_{\mathrm{R}, \mathrm{L}} \int_{\eta_{i}}^{\eta} d \eta\{\epsilon \mathcal{Y}[E](\eta)-2 \gamma \zeta \mathcal{Y}[\Gamma Z](\eta)\}
$$

When $\gamma \ll 1$, i.e., $k_{0}$ is very much greater than the Hubble constant $H_{0}$, the rescaled frequency $\left|y_{0}\right| \sim 1$. In this limit we can use integration by parts to find an asymptotic expansion for $\mathcal{Y}[g]$ :

$$
\mathcal{Y}[g](\eta) \sim \frac{i e^{2 i \phi_{0}(\eta)}}{2}\left[e^{-2 i \phi_{0}} \sum_{\ell=0}^{n}\left(\frac{1}{2 i \kappa}\right)^{\ell}\left(\frac{1}{y_{0}} \frac{d}{d \eta}\right)^{\ell} g\right]_{\eta_{i}}^{\eta}+\mathcal{O}\left(\frac{1}{2 i \kappa}\right)^{n+1}
$$

In the next section we explore the observational consequences of gravitational wave propagation in Chern-Simons gravity.

\section{OBSERVATIONAL CONSEQUENCES}

\section{A. Birefringence in a Matter-Dominated Cosmology}

Current and proposed ground-based gravitational wave detectors are sensitive to gravitational waves in the $10 \mathrm{~Hz}$ $-1 \mathrm{KHz}$ band [32, 33, 34, 35]. Detectable sources in this band are expected to have redshifts $z \lesssim 1$. Space-based gravitational wave detectors like LISA [13] will be sensitive to gravitational waves in the $0.1-100 \mathrm{mHz}$ band and, in this band, be sensitive enough to observe the gravitational waves from the inspiral of several $\sim 10^{6} \mathrm{M}_{\odot}$ black hole binary systems at $z \lesssim 30$ : i.e., anywhere in the universe they are expected [36, 37]. For sources in the band of these detectors

$$
\gamma=3.7 \times 10^{-19}\left(\frac{h_{100}}{0.72}\right)\left(\frac{1 \mathrm{~Hz}}{k c / 2 \pi}\right) \ll 1
$$

where

$$
h_{100}=\frac{H_{0}}{100 \mathrm{~km} \mathrm{~s}^{-1} \mathrm{Mpc}^{-1}} .
$$

Additionally, for redshitfs $z \lesssim 30$ the universe is well described by a matter-dominated FRW cosmological model. In this section we evaluate the effect that the Chern-Simons corrections described above have on propagation of gravitational plane-waves through a matter-dominated FRW model.

In a matter-dominated FRW model the scale factor $a(t)$ satisfies [31]

$$
\frac{a(\eta)}{a_{0}}=\eta^{2}=\frac{1}{1+z}
$$


where, by convention, $\eta=1$ at the present epoch. In this model and with this convention

$$
\begin{aligned}
& \mathcal{H}=\frac{2}{\eta}=2 \sqrt{1+z} \quad \text { and } \quad \mathcal{H}_{0}=2 \text {, } \\
& \gamma=\frac{2}{\kappa} \quad \text { and } \quad \Gamma=\eta^{-1}=\sqrt{1+z}, \\
& \delta=-\frac{2}{\kappa^{2}} \quad \text { and } \quad \Delta=\eta^{-2}=1+z, \\
& \epsilon=\frac{H_{0}^{2}}{4} \theta_{0}^{\prime \prime} \quad \text { and } \quad E=\frac{1}{\eta^{4}} \frac{\theta^{\prime \prime}}{\theta_{0}^{\prime \prime}}, \\
& \zeta=\frac{H_{0} k}{2} \theta_{0}^{\prime} \quad \text { and } \quad Z=\frac{1}{\eta^{4}} \frac{\theta^{\prime}}{\theta_{0}^{\prime}} \text {, }
\end{aligned}
$$

Additionally,

$$
\begin{aligned}
E & =\frac{1}{\eta^{4}} \frac{\theta^{\prime \prime}}{\theta_{0}^{\prime \prime}} \\
Z & =\frac{1}{\eta^{4}} \frac{\theta^{\prime}}{\theta_{0}^{\prime}} \\
a_{0} & =\frac{\mathcal{H}_{0}}{H_{0}}=\frac{2}{H_{0}} .
\end{aligned}
$$

With the $\gamma$ and $\Gamma$ parameters for a matter-dominated FRW cosmological model, the unperturbed equation for $y_{0}$ has solutions of the form

$$
y_{0}=-\frac{i}{\kappa \eta} \frac{\left(1+C \kappa \eta-\kappa^{2} \eta^{2}\right) \cos (\kappa \eta)-\left(C-\kappa \eta-C \kappa^{2} \eta^{2}\right) \sin (\kappa \eta)}{(1+C \kappa \eta) \cos (\kappa \eta)-(C-\kappa \eta) \sin (\kappa \eta)}
$$

where $C$ is a constant of the integration. In the limit of large $\kappa$ the evolution of $\kappa$ should decouple from the universal expansion; thus, we are led to choose $C= \pm i$, which eliminates the oscillatory terms in our general solution for $y_{0}$ :

$$
y_{0}=\frac{ \pm \kappa^{3} \eta^{3}-i}{\kappa \eta\left(1+\kappa^{2} \eta^{2}\right)}
$$

Consistent with our ansatz [cf. Eq. (2.13)] we choose the solution with positive $\Re\left(y_{0}\right)$ : i.e., $C=+i$. Solving this equation for the phase $\phi_{0}^{\prime}=\kappa y_{0}$ we find

$$
\begin{aligned}
\Delta \phi_{0}(\eta) & =\phi_{0}(1)-\phi_{0}(\eta) \\
& =\left[\kappa(1-\eta)-\arctan \frac{\kappa(1-\eta)}{1+\kappa^{2} \eta}\right]-\frac{i}{2} \ln \left[\frac{1+\kappa^{2} \eta^{2}}{\eta^{2}\left(1+\kappa^{2}\right)}\right] \\
& =\left[\frac{2}{\gamma} \frac{\sqrt{1+z}-1}{\sqrt{1+z}}-\arctan \frac{2 \gamma(\sqrt{1+z}-1)}{4+\gamma^{2} \sqrt{1+z}}\right]-\frac{i}{2} \ln \left[1+\frac{\gamma^{2} z}{4+\gamma^{2}}\right] .
\end{aligned}
$$

In the absence of the Chern-Simons correction an observer at rest with respect to slices of (cosmological) homogeneity will observe a passing gravitational plane-wave to undergo a change in phase $\Delta \phi_{0}(\eta)$ between cosmological time $\eta$ and the present epoch.

With $y_{0}$ and equations (2.23) we can evaluate the Chern-Simons contribution to the phase change owing to propagation from cosmological time $\eta_{0}$. Making use of the asymptotic expansions for $y_{0,1}$ and $y_{1,0}$ [Eq. (2.25)] we find

$$
\begin{aligned}
y_{0,1(\mathrm{R}, \mathrm{L})} & \sim \frac{i \lambda_{\mathrm{R}, \mathrm{L}}}{2}\left[E(\eta)-E\left(\eta_{0}\right) e^{-2 i\left[\Delta \phi_{0}\left(\eta_{0}\right)-\Delta \phi_{0}(\eta)\right]}\right]+\mathcal{O}(\gamma) \\
y_{1,0(\mathrm{R}, \mathrm{L})} & \sim-i \lambda_{\mathrm{R}, \mathrm{L}}\left[\Gamma(\eta) Z(\eta)-\Gamma\left(\eta_{0}\right) Z\left(\eta_{0}\right) e^{-2 i\left[\Delta \phi_{0}\left(\eta_{0}\right)-\Delta \phi_{0}(\eta)\right]}\right]+\mathcal{O}(\gamma),
\end{aligned}
$$

which may be integrated to find $\Delta \phi_{1(\mathrm{R}, \mathrm{L})}$ :

$$
\begin{aligned}
\Delta \phi_{1(\mathrm{R}, \mathrm{L})} & =i \lambda_{\mathrm{R}, \mathrm{L}} \frac{\kappa}{2} \int_{\eta}^{1}\left[\epsilon \frac{1}{\eta^{4}} \frac{\theta^{\prime \prime}(\eta)}{\theta_{0}^{\prime \prime}}-2 \gamma \zeta \frac{1}{\eta^{5}} \frac{\theta^{\prime}(\eta)}{\theta_{0}^{\prime}}\right] d \eta+\mathcal{O}(\gamma), \\
& =i \lambda_{\mathrm{R}, \mathrm{L}} \frac{k_{0}}{H_{0}} \xi(\eta) .
\end{aligned}
$$


It is convenient to rewrite $\xi$ as a function of $z$ :

$$
\xi(z)=\alpha A(z)+\beta B(z)
$$

where

$$
\begin{aligned}
A(z) & =\int_{0}^{z} d z(1+z)^{5 / 2} \frac{d \theta / d z}{(d \theta / d z)_{0}} \\
B(z) & =\int_{0}^{z} d z(1+z)^{7 / 2} \frac{d^{2} \theta / d z^{2}}{\left(d^{2} \theta / d z^{2}\right)_{0}} \\
\alpha & =-\gamma \zeta+\frac{3 \epsilon}{2} \frac{(d \theta / d z)_{0}}{2\left(d^{2} \theta / d z^{2}\right)_{0}+3(d \theta / d z)_{0}}, \\
\beta & =\frac{\epsilon\left(d^{2} \theta / d z^{2}\right)_{0}}{2\left(d^{2} \theta / d z^{2}\right)_{0}+3(d \theta / d z)_{0}},
\end{aligned}
$$

and the subscript zero denotes present-day values of the subscripted quantities. The leading order Chern-Simons correction to the accumulated phase is thus pure imaginary, corresponding to an attenuation of one circular polarization state and an equal amplification of the other. The attenuation/amplification is linearly dependent on the wavenumber. The function $\xi(z)$ may be thought of as a "form-factor" that probes the past history of the coupling $\theta$.

\section{B. Binary Inspiral at Cosmological Distances}

The proposed LISA gravitational wave detector is capable of observing coalescing binary black hole systems at cosmological distances: for example, the gravitational waves associated with a pair of $10^{6} \mathrm{M}_{\odot}$ black holes will be observable at redshifts $z$ approaching 30. Over the year leading up to the merger of two such black holes the binary's period will decrease by two orders of magnitude, leading to a corresponding decrease in the radiation wavelength and increase in the magnitude of the Chern-Simons correction. The time-dependent relationship between the radiation amplitude in the two polarization states thus carries with it the signature of Chern-Simons gravity and can be used to characterize the functional $\theta$ that describes the Chern-Simons correction to classical General Relativity.

To calculate the signature left by the Chern-Simons correction on the gravitational waves from a coalescing binary system at redshift $z$, we begin with the radiation near the source. Treating, as before, the Chern-Simons correction as a perturbation, the quadrupole approximation to the radiation from the binary system in the neighborhood of the source is given by

$$
\begin{aligned}
\widehat{\mathbf{h}} & =\Re\left[\widehat{h}_{+} e_{+}+\widehat{h}_{\times} e_{\times}\right] \\
\widehat{h}_{+} & =\frac{2 \widehat{\mathcal{M}}}{d}\left[1+\hat{\chi}^{2}\right][\widehat{\mathcal{M}} \hat{k}(\hat{t}) / 2]^{2 / 3} \exp [-i(\hat{\Phi}(\hat{t})-\hat{k}(\hat{t}) d)], \\
\widehat{h}_{\times} & =\frac{4 i \widehat{\mathcal{M}}}{d} \hat{\chi}[\widehat{\mathcal{M}} \hat{k}(\hat{t}) / 2]^{2 / 3} \exp [-i(\hat{\Phi}(\hat{t})-\hat{k}(\hat{t}) d)],
\end{aligned}
$$

where $d$ is the proper distance to the source and

$$
\begin{aligned}
& \hat{\Phi}(\hat{t})=-2\left(\frac{\hat{T}-\hat{t}}{5 \widehat{\mathcal{M}}}\right)^{5 / 8}+\hat{\delta} \\
& \hat{k}(\hat{t})=\frac{2}{\widehat{\mathcal{M}}}\left(\frac{5}{256} \frac{\widehat{\mathcal{M}}}{\hat{T}-\hat{t}}\right)^{3 / 8} .
\end{aligned}
$$

The constants $\hat{T}$ and $\hat{\delta}$, which determine when coalescence occurs and the phase of the gravitational wave signal at some fiducial instant, are set by initial conditions. The quantities $\widehat{\mathcal{M}}$ and $\hat{\chi}$ are constants that depend on the binary system's component masses $\left(m_{1}, m_{2}\right)$ and orientation with respect to the observer:

$$
\begin{aligned}
\hat{\chi} & =\left(\begin{array}{l}
\text { cosine-angle between the orbital angular } \\
\text { momentum and the observer line-of-sight }
\end{array}\right) \\
\widehat{\mathcal{M}} & =\frac{m_{1}^{3 / 5} m_{2}^{3 / 5}}{\left(m_{1}+m_{2}\right)^{1 / 5}}=(\text { "chirp" mass })
\end{aligned}
$$


We "hat" all these quantities to remind us that, as expressed above, they are appropriate descriptions only in the neighborhood of the source where the Chern-Simons and cosmological corrections to the propagation of the waves may be neglected.

To describe the radiation after it has propagated to the detector we first describe the near-source radiation in terms of circular polarization states

$$
\begin{aligned}
\widehat{\mathbf{h}} & =\Re\left[\widehat{h}_{R} e_{R}+\widehat{h}_{L} e_{L}\right], \\
\widehat{h}_{R, L} & =\sqrt{2} \frac{\widehat{\mathcal{M}}}{d}\left(\frac{\widehat{\mathcal{M}} k}{2}\right)^{2 / 3}\left(1+\lambda_{R, L} \hat{\chi}\right)^{2} \exp [-i(\hat{\Phi}(\hat{t})-\hat{k}(\hat{t}) d)] .
\end{aligned}
$$

We are interested in the radiation incident on our detector today $(z=0, \eta=1)$ from a source at redshift $z$. Matching the near-source description of the radiation [Eq. (3.13)) to our ansatz [Eq. (2.13)] we find the description of the radiation after propagating to the detector from a redshift $z$ :

$$
\begin{aligned}
\mathbf{h} & =\Re\left[\widehat{h}_{R} e_{R}+\widehat{h}_{L} e_{L}\right], \\
h_{\mathrm{R}, \mathrm{L}} & =\sqrt{2} \frac{\mathcal{M}}{d_{L}}\left(\frac{\mathcal{M} k_{0}}{2}\right)^{2 / 3}\left(1+\lambda_{R, L} \hat{\chi}\right)^{2} \exp \left[-i\left(\Phi_{0}(t)-\kappa(1-\eta)+\Delta \phi_{0}(t)+\Delta \phi_{1(\mathrm{R}, \mathrm{L})}(t)\right)\right]
\end{aligned}
$$

where

$$
\begin{aligned}
\Phi_{0}(t) & =-2\left(\frac{T-t}{5 \mathcal{M}}\right)^{5 / 8}+\delta \\
k_{0}(t)= & \frac{2}{\mathcal{M}}\left(\frac{5}{256} \frac{\mathcal{M}}{T-t}\right)^{3 / 8} \\
d_{L}= & a_{0} \eta(1+z)=(\text { Luminosity distance to source }) \\
\mathcal{M} & =(1+z) \widehat{\mathcal{M}} \\
\Delta \phi_{0}= & \frac{2(\sqrt{1+z}-1)}{H_{0} \sqrt{1+z}} k_{0}(t)-\arctan 2 \gamma(t) \frac{\sqrt{1+z}-1}{4+\sqrt{1+z} \gamma^{2}(t)} \\
& \quad-\frac{i}{2} \ln \left[1+\gamma^{2}(t) \frac{z}{4+\gamma^{2}(t)}\right] \\
\gamma(t)= & \frac{H_{0}}{k_{0}(t)},
\end{aligned}
$$

and $\Delta \phi_{1(\mathrm{R}, \mathrm{L})}$ given by equations (3.8) above. Here $t$ is proper time as measured by a detector at rest with respect to the cosmological fluid at the present epoch $(\eta=1), k_{0}(t)$ is the instantaneous wavenumber of the wavefront passing the detector at observer time $t$, and $T$ and $\delta$ are, as before, constants of the integration. The correction $\Delta \phi_{0}$, which is the same for all polarizations, embodies $\mathcal{O}\left(k_{0} / H_{0}\right)$ corrections to the wave phase owing to the wave propagation through the time-dependent cosmological background. The correction $\Delta \phi_{1(\mathrm{R}, \mathrm{L})}$ is of opposite character for the two polarization states and embodies the (first-order) corrections to wave propagation owing to the Chern-Simons corrections to the Einstein Field Equations.

Focus attention on the argument of the exponential in equation (3.14b). The term $\kappa(1-\eta)$ cancels the first term in equation (3.14g) for $\Delta \phi_{0}$, leading to

$$
h_{\mathrm{R}, \mathrm{L}}=\sqrt{2} \frac{\mathcal{M}}{d_{L}}\left(\frac{\mathcal{M} k_{0}}{2}\right)^{2 / 3}\left(1+\lambda_{R, L} \hat{\chi}\right)^{2} \exp \left[-i\left(\Phi_{0}(t)-\frac{\gamma(t)}{2}(\sqrt{1+z}-1)+\Delta \phi_{1(\mathrm{R}, \mathrm{L})}(t)\right)\right]
$$

The observational effect of the Chern-Simons is readily identified by looking at the ratio of the polarization amplitudes $h_{R}$ and $h_{L}$ :

$$
\begin{aligned}
\frac{h_{\mathrm{R}}}{h_{\mathrm{L}}} & =\frac{(1+\hat{\chi})}{(1-\hat{\chi})} \exp \left[\frac{2 k(t) \xi(z)}{H_{0}}\right] \\
& =\frac{1+x}{1-x}
\end{aligned}
$$


where $\xi$ is given by equations (3.8) and $x$ may be interpreted as the apparent inclination cosine-angle. The effect of the Chern-Simons correction on gravitational wave propagation is to confound the identification between polarization amplitude ratios and binary orbit inclination cosine-angle. In the same way that we say that the curvature of spacetime "bends" light passing close to strongly gravitating body we may say that the effect of the Chern-Simons correction is to "rotate" the apparent inclination angle of the binary system's orbital angular momentum axis either toward or away from us.

\section{DISCUSSION}

\section{A. What can be measured?}

Over the course of a year-long observation the LISA spacecraft constellation will measure the radiation in both polarizations of an incident gravitational wave train associated with an inspiraling coalescing binary system. The relative amplitude of the two polarization will be determined by the orientation of the binary systems orbital plane to the observer line-of-sight and the form factor $\xi(z)$. A non-vanishing $\xi$ leads to a time-varying apparent inclination angle that, by nature of its time dependence, can (in principle) be measured directly from the apparent inclination angle's time variation.

Other properties of an inspiraling binary can lead to an evolution of the (apparent) inclination cos-angle. Spin-orbit coupling leads to a real precession of the binary's orbital plane and a corresponding time-dependence in the actual inclination cos-angle $\hat{\chi}$. Referring to equation (3.14b), it is apparent that for small $|\hat{\chi}| \sim 0$ an incremental change $\mu$ in $\hat{\chi}$ will lead to changes in $h_{\mathrm{R}, \mathrm{L}}$ that are indistinguishable from an increment in $x$ associated with $\xi$. Following Vecchio [38, eq. 27-31] we note that, at first non-vanishing post-Newtonian order, spin-orbit interactions in an inspiraling binary system lead to

$$
\left(\frac{d \hat{\chi}}{d t}\right)_{\text {so }} \propto k_{0}^{2 / 3}(t)
$$

This is a different dependence on $k_{0}$ than the $\mathcal{O}\left(k_{0}\right)$ dependence associated with $\xi$. Thus, it remains in principle possible to distinguish the signature of Chern-Simons gravity in the signal from cosmologically distant coalescing binary black hole systems. The accuracy with which such a measurement can be made is the topic of the next subsection.

\section{B. How accurately can $\xi$ be measured?}

The most general astrophysical black hole binary system can be described by eleven independent parameters, which may be counted as two component masses; component spins and their orientation (six parameters); orbital eccentricity; orbital phase; and a a reference time when the phase, spins and eccentricity are measured. The gravitational wave signal in any particular polarization will depend on the description of the binary and six additional parameters that describe the binary's orientation with respect to the detector. These six additional parameters may be counted as orbital plane orientation (two angles); source location with respect to the detector (distance and two position angles); orbit orientation in orbital plane (one angle) [61]. To these seventeen parameters we now add $\xi$, which describes the effect of propagation through the birefringent Chern-Simons spacetime, for a total of eighteen parameters that are required to describe the signal from a coalescing binary system.

To-date, all analyses of expected parameter estimation errors have been made with under a set of approximations that focus attention on the measurement of component masses, source location (both distance and angular position), and the expected time of binary coalescence. Even the most sophisticated of these analysis ignore all but the leadingorder contribution to the gravitational wave signal amplitude at twice the orbital frequency [62] and assume that the orbital eccentricity is known to vanish. These approximations are quite appropriate for their purpose (estimation of component masses, source location and expected time of coalescence); however, by ignoring all but the leading order contribution to the signal magnitude they are inadequate starting points for exploring the accuracy with which $\xi$, which affects only the signal amplitude in the different polarizations, can be bounded [63]. Evaluating and presenting the errors associated with the measurement of $\xi$ via a full co-variance matrix analysis is thus a formidable enterprise, to be addressed in a future work.

Nevertheless, through a series of plausible approximations it is possible to make a crude estimate of the accuracy with which $\xi$ can be determined. To begin, assume we have two gravitational wave detectors such that, via a linear 
combination of observations made at each, we can can synthesize two other detectors with one exclusively sensitive to $h_{\mathrm{R}}$ and and one exclusively sensitive to $h_{\mathrm{L}}$. Write the scalar detector response of each of these detectors as

$$
m_{\mathrm{R}, \mathrm{L}}(t)=\exp \left[\mu_{\mathrm{R}, \mathrm{L}}(t)+i \psi_{\mathrm{R}, \mathrm{L}}(t)\right],
$$

for real $\mu_{\mathrm{R}, \mathrm{L}}$ and $\psi_{\mathrm{R}, \mathrm{L}}$. Next, note that the parameters that describe a coalescing binary system can be divided into two groups: those that principally affect only the signal amplitude (i.e., $\mu(t))$ and those that affect only or principally the real part of the signal phase (i.e., $\psi(t)$ ). The first group includes distance, source orientation with respect to the observer line-of-sight, and $\xi$. The second group includes the orbital phase, sky location (through its affect on the Doppler correction to the signal phase as the detector orbits about the sun), the instantaneous binary period at some fiducial moment, and the parameters associated with spin and orbital angular momentum [64]. If we approximate each detectors' noise as white with one-sided noise power spectral density $S_{0}$ then the elements of the inverse covariance matrix $\boldsymbol{\Gamma}$ — the so-called Fisher matrix — are given by [39, 40]

$$
\Gamma_{i j}=\sum_{k=\mathrm{R}, \mathrm{L}} \frac{2}{S_{0}} \int_{t_{i}}^{t_{f}} \Re\left(\frac{\partial m_{k}}{\partial x^{i}}\right) \Re\left(\frac{\partial m_{k}}{\partial x^{j}}\right) d t
$$

where the integration is over the observation period $\left(t_{i}, t_{f}\right)$ and the $x^{i}$ are the parameters that characterize the incident gravitational wave, which we have divided into two groups. Matrix elements $\Gamma_{i j}$ where $x^{i}$ and $x^{j}$ belong to different groups will be much smaller than elements where $x^{i}$ and $x^{j}$ belong to the same group. Setting the cross-group elements to zero we obtain an approximate $\boldsymbol{\Gamma}$ that is block diagonal, with one block corresponding to $\Gamma_{i j}$ with $\left(x^{i}, x^{j}\right)$ drawn from the first group and the other block corresponding to $\Gamma_{i j}$ with $\left(x^{i}, x^{j}\right)$ drawn from the second group. Estimation uncertainties of parameters in either group can now be determined independently of the parameters in the other group.

Focus attention now on those parameters that affect only $\mu(t)$, the signals amplitude evolution. The leading order dependence of the amplitude $\left|h_{\mathrm{R}, \mathrm{L}}\right|$ on the binary systems parameters is given by

$$
A_{\mathrm{R}, \mathrm{L}}=\left|h_{\mathrm{R}, \mathrm{L}}\right|=\left(1+\lambda_{\mathrm{R}, \mathrm{L}} \hat{\chi}_{0}\right)^{2} \frac{2 \mathcal{M}}{d_{L}}\left[\frac{k_{0}(t) \mathcal{M}}{2}\right]^{2 / 3} \exp \left(\lambda_{\mathrm{R}, \mathrm{L}} \xi \frac{k_{0}(t)}{H_{0}}\right)
$$

where $\mathcal{M}$ is assumed known. Setting aside the antenna pattern factors associated with the projection of the signal onto the LISA detector (which depend only on the known source sky position and the LISA orbital ephemeris), assuming that there is no real precession in the binary system under observation (i.e., $\hat{\chi}_{0}$ ), and that $k(t)$ is given by equation (3.11) the inverse of the covariance matrix - the so-called Fisher matrix, $\boldsymbol{\Gamma}$ - associated with the amplitude measurements is a symmetric $3 \times 3$ matrix with elements

$$
\begin{aligned}
\Gamma_{\mathcal{D D}} & =\frac{1}{S_{0}} \int_{t_{i}}^{t_{f}}\left(A_{\mathrm{R}}^{2}+A_{\mathrm{L}}^{2}\right) d t \simeq 8\left(1+6 \hat{\chi}_{0}^{2}+\hat{\chi}_{0}^{4}\right)\left(\frac{\mathcal{M}}{d_{L}}\right)^{2} \mathcal{I}+\mathcal{O}(\xi) \\
\Gamma_{\mathcal{D} \hat{\chi}_{0}} & =\frac{1}{S_{0}} \int_{t_{i}}^{t_{f}}\left[\frac{2}{1-\hat{\chi}_{0}} A_{\mathrm{L}}^{2}-\frac{2}{1+\hat{\chi}_{0}} A_{\mathrm{R}}^{2}\right] d t \simeq-16 \hat{\chi}_{0}\left(3+\hat{\chi}_{0}^{2}\right)\left(\frac{\mathcal{M}}{d_{L}}\right)^{2} \mathcal{I}+\mathcal{O}(\xi) \\
\Gamma_{\mathcal{D} \xi} & =\frac{1}{S_{0}} \int_{t_{i}}^{t_{f}} \frac{k_{0}(t)}{H_{0}}\left(A_{\mathrm{L}}^{2}-A_{\mathrm{R}}^{2}\right) d t \simeq-\frac{64 \hat{\chi}_{0}\left(1+\hat{\chi}_{0}^{2}\right)}{\mathcal{M} H_{0}}\left(\frac{\mathcal{M}}{d_{L}}\right)^{2} \mathcal{J}+\mathcal{O}(\xi) \\
\Gamma_{\hat{\chi}_{0} \hat{\chi}_{0}} & =\frac{1}{S_{0}} \int_{t_{i}}^{t_{f}}\left[\frac{4 A_{\mathrm{R}}^{2}}{\left(1+\hat{\chi}_{0}\right)^{2}}+\frac{4 A_{\mathrm{L}}^{2}}{\left(1-\hat{\chi}_{0}\right)^{2}}\right] d t \simeq 32\left(1+\hat{\chi}_{0}^{2}\right)\left(\frac{\mathcal{M}}{d_{L}}\right)^{2} \mathcal{I}+\mathcal{O}(\xi) \\
\Gamma_{\hat{\chi}_{0} \xi} & =\frac{1}{S_{0}} \int_{t_{i}}^{t_{f}} \frac{2 k_{0}(t)}{H_{0}}\left[\frac{A_{\mathrm{R}}^{2}}{1+\hat{\chi}_{0}}+\frac{A_{\mathrm{L}}^{2}}{1-\hat{\chi}_{0}}\right] d t \simeq \frac{32\left(1+3 \hat{\chi}_{0}^{2}\right)}{\mathcal{M} H_{0}}\left(\frac{\mathcal{M}}{d_{L}}\right)^{2} \mathcal{J}+\mathcal{O}(\xi) \\
\Gamma_{\xi \xi} & =\frac{1}{S_{0}} \int_{t_{i}}^{t_{f}} \frac{k_{0}^{2}(t)}{H_{0}^{2}}\left(A_{\mathrm{R}}^{2}+A_{\mathrm{L}}^{2}\right) d t \simeq \frac{32\left(1+6 \hat{\chi}_{0}^{2}+\hat{\chi}_{0}^{4}\right)}{\left(\mathcal{M} H_{0}\right)^{2}}\left(\frac{\mathcal{M}}{d_{L}}\right)^{2} \mathcal{K}+\mathcal{O}(\xi)
\end{aligned}
$$


where

$$
\begin{aligned}
\mathcal{D} & =\ln d_{L} \\
\mathcal{I} & =\int_{t_{i}}^{t_{f}}\left(\frac{k_{0}(t) \mathcal{M}}{2}\right)^{4 / 3} \frac{d t}{S_{0}}=\frac{5}{192} \int_{k_{\min }}^{k_{\max }}\left(\frac{k \mathcal{M}}{2}\right)^{-7 / 3} \frac{\mathcal{M}^{2} d k}{S_{0}}=-\left.\frac{2^{1 / 3} \mathcal{M}}{S_{0}} \frac{5}{64}(k \mathcal{M})^{-4 / 3}\right|_{k_{\min }} ^{k_{\max }} \\
\mathcal{J} & =\int_{t_{i}}^{t_{f}}\left(\frac{k_{0}(t) \mathcal{M}}{2}\right)^{7 / 3} \frac{d t}{S_{0}}=\frac{5}{192} \int_{k_{\min }}^{k_{\max }}\left(\frac{k \mathcal{M}}{2}\right)^{-4 / 3} \frac{\mathcal{M}^{2} d k}{S_{0}}=-\left.\frac{2^{1 / 3} \mathcal{M}}{S_{0}} \frac{5}{32}(k \mathcal{M})^{-1 / 3}\right|_{k_{\min }} ^{k_{\max }} \\
\mathcal{K} & =\int_{t_{i}}^{t_{f}}\left(\frac{k_{0}(t) \mathcal{M}}{2}\right)^{10 / 3} \frac{d t}{S_{0}}=\frac{5}{192} \int_{k_{\min }}^{k_{\max }}\left(\frac{k \mathcal{M}}{2}\right)^{-1 / 3} \frac{\mathcal{M}^{2} d k}{S_{0}}=\left.\frac{2^{1 / 3} \mathcal{M}}{S_{0}} \frac{5}{128}(k \mathcal{M})^{2 / 3}\right|_{k_{\min }} ^{k_{\max }}
\end{aligned}
$$

and we have taken advantage of the fact that for inspiraling compact binary systems in the quadrupole approximation $k(t)$ is monotonic in $t$ to re-express the integrals over the interval $\left(t_{i}, t_{f}\right)$ as integrals over $\left[k\left(t_{i}\right), k\left(t_{f}\right)\right]=\left(k_{\min }, k_{\max }\right)$. In the particular case of a binary seen plane-on $\left(\hat{\chi}_{0}=0\right)$, the $\left(\mathcal{D} \hat{\chi}_{0}\right)$ and $(\mathcal{D} \xi)$ blocks of $\boldsymbol{\Gamma}$ are diagonal, leading to

$$
\begin{aligned}
\nu_{\hat{\chi}_{0} \hat{\chi}_{0}} & =\frac{1}{4 \rho^{2}} \frac{\mathcal{K} \mathcal{I}}{\mathcal{I} \mathcal{K}-\mathcal{J}^{2}} \\
\nu_{\xi \xi} & =\frac{\left(\mathcal{M} H_{0}\right)^{2}}{4 \rho^{2}} \frac{\mathcal{I}^{2}}{\mathcal{I} \mathcal{K}-\mathcal{J}^{2}}
\end{aligned}
$$

where $\nu_{i j}$ is the ensemble average co-variance

$$
\nu_{i j}=\overline{\left(x^{i}-\overline{x_{i}}\right)\left(x^{j}-\overline{x_{j}}\right)}=\left(\boldsymbol{\Gamma}^{-1}\right)_{i j}
$$

and we have expressed the $\nu_{i j}$ in terms of the ensemble average amplitude-squared signal-to-noise ratio $\rho^{2}$

$$
\rho^{2}=\frac{1}{S_{0}} \int_{t_{i}}^{t_{f}} d t\left(A_{R}^{2}+A_{L}^{2}\right)
$$

Focus attention on a binary system of two black holes at redshift $z$, each with mass $M=10^{6} M_{\odot}(1+z)^{-1}$. Over the final year before coalescence the radiation wavelength $2 \pi / k$ observed at the detector will range from $c\left(10^{-4} \mathrm{~Hz}^{-1}\right.$ to $c\left(10^{-2} \mathrm{~Hz}\right)^{-1}$. For such a system,

$$
\begin{aligned}
\rho & =\frac{10^{5} h_{100}}{1+z-\sqrt{1+z}}\left(\frac{10^{-40} \mathrm{~Hz}^{-1}}{S_{0}}\right)^{1 / 2} \\
\nu_{\xi \xi} & =3.1 \times 10^{-40}\left(\frac{S_{0}}{10^{-40} \mathrm{~Hz}^{-1}}\right)(1+z-\sqrt{1+z})^{2}
\end{aligned}
$$

Observation of binary systems like these at $z=15$ by LISA will be capable of placing a "1-sigma" upper bound on $\xi$ of order $10^{-19}$.

\section{How large might $\xi$ be?}

To estimate $\xi$ [cf. Eq. (3.8c)] we must invoke a theoretical model for the functional $\theta[\phi(z)]$. As described in the introduction, perturbative string theory requires a Chern-Simons correction to the Einstein-Hilbert action [65]. Here we describe a different mechanism, that can also lead to the presence of a Chern-Simons correction. Consider the back-reaction of a $\mathcal{N}=1$ supersymmetric Yang-Mills theory in a curved background (cf. [41, Appendix A]) with action

$$
S_{\mathrm{CS}}=\frac{1}{16 \pi} \int d^{4} x \mathcal{F}_{1}(\mathrm{~S})\left(\mathrm{R}^{\star} \mathrm{R}\right),
$$

where $S$ is the glueball superfield and $\mathcal{F}_{1}(\mathrm{~S})$, which plays the role of $\theta$ in equation (2.1), can be exactly evaluated by using perturbative matrix model technology developed in [42]. Within this Yang-Mills framework, $\theta$ is a functional 
$\theta[\varphi]$ of some pseudo-scalar field $\varphi$, the gravitational axion, that depends only on conformal time [12]. The functional $\theta[\varphi]$ can be expressed as

$$
\theta[\varphi]=\frac{\mathcal{N} \ell_{\mathrm{Pl}}^{2}}{2 \pi} \frac{\varphi}{M_{\mathrm{Pl}}}
$$

where $\ell_{\mathrm{Pl}}=\left(\hbar G / c^{3}\right)^{1 / 2}$ is the Planck length, $M_{\mathrm{Pl}}=\left(\ell_{\mathrm{Pl}} \sqrt{8 \pi}\right)^{-1}$ is the reduced Planck mass and $\mathcal{N}$ is a dimensionless constant. Through use of the low-energy effective 4-dimensional heterotic string action the constant $\mathcal{N}$ can be evaluated in terms of the ten-dimensional fundamental string energy scale $M_{\mathrm{s}}$ and the dimensionless string coupling $g_{\mathrm{s}}$,

$$
\mathcal{N}=\left(\frac{M_{\mathrm{Pl}}}{M_{\mathrm{s}}}\right)^{2} \frac{1}{\sqrt{g_{\mathrm{s}}}}
$$

leading to

$$
\theta[\varphi]=\frac{1}{16 \pi^{2} M_{\mathrm{Pl}}^{2}} \frac{\varphi}{M_{\mathrm{s}}} \frac{M_{\mathrm{Pl}}}{M_{\mathrm{s}} \sqrt{g}_{\mathrm{s}}}
$$

Assuming that $\varphi$, which has units of inverse length, evolves with the Hubble parameter $H \propto \eta^{-3}$ we have

$$
A(z)=B(z)=-\frac{2}{11}\left[(1+z)^{11 / 2}-1\right]
$$

and

$$
\xi=-\frac{1}{11}\left[(1+z)^{11 / 2}-1\right](\epsilon-2 \gamma \zeta)
$$

with $(\epsilon-\gamma \zeta)$ of the order of

$$
\epsilon-2 \gamma \zeta \simeq \frac{\left(1.8+3.5 h_{100}^{2}\right) \times 10^{-120}}{\sqrt{g_{\mathrm{s}}}}\left(\frac{\varphi_{0}}{M_{\mathrm{s}}}\right)\left(\frac{10^{16} \mathrm{GeV}}{M_{\mathrm{s}}}\right) .
$$

The size of $\xi$ thus depends on the present value of the field $\varphi_{0}$, the fundamental string energy scale $M_{s}$ and the string coupling $g_{\mathrm{s}}$, none of which are constrained by present-day theory.

The lesson to draw from the discussion of this scenario is that the magnitude of any Chern-Simons correction depends strongly on the external theoretical framework that prescribes the functional $\theta[\varphi]$. For non-vanishing string coupling in the perturbative string theory scenario the Chern-Simons correction seems undetectable owing to the Planck scale suppression of the decay constant of the universal gravitational axion field $\varphi$. However, this model and the associated expected scale of $\epsilon-2 \gamma \zeta$ applies only to the perturbative sector of string theory and, in particular, when RamondRamond charges are turned off. If present these additional degrees of freedom do couple and source the Chern-Simons correction, leading to a larger decay constant (e.g, D3 branes always excite the Chern-Simons interaction in four dimensions). In a recent work, Svrcek [43] noted that, due to non-perturbative gravitational instanton corrections, the Chern-Simons coupling in the non-perturbative sector is currently incalculable. Even within the perturbative framework there are theoretical frameworks where $\xi$ could become significant: e.g., if the string coupling $g_{\mathrm{s}}$ vanishes at late times [44, 45, 46, 47, 48, 49, 50, 51, 52, 53, 54]. Therefore, within the full string theory framework, a larger coupling, which would push the stringy Chern-Simons correction into the observational window, is not excluded and bounding it places a constraint on string theory motivated corrections to classical general relativity.

Setting aside string theory for a moment, other theoretical frameworks lead to a Chern-Simons correction to the Einstein-Hilbert action. Jackiw and Pi [8] showed that the embedding of the 3-dimensional Chern-Simons action into 4dimensional General Relativity leads to Chern-Simons gravity as described by equation (2.1). Quantum mechanically, the Chern-Simons correction encodes information about the Immirzi parameter in loop quantum gravity and is also related to torsion. In this case, the Chern-Simons term is required to ensure invariance under large gauge transformations [3]. In both these classical and quantum mechanical scenarios the coupling of the Chern-Simons term to the Einstein-Hilbert action is theoretically unconstrained and is consistent with a coupling of order unity. Observations of gravitational waves from inspiraling binary black hole systems can thus be used to probe the strong gravity sector of quantum gravity. 


\section{CONCLUSIONS}

Chern-Simons type corrections to the Einstein-Hilbert action are strongly motivated by string theory, loop quantum gravity, and other scenarios. In all cases these corrections lead to an amplitude birefringence for gravitational wave propagating through space time. We have evaluated the correction to the gravitational wave amplitude for waves propagating over cosmological distances in a matter-dominated Friedmann-Robertson-Walker cosmology. In the case of the gravitational waves from inspiraling binary black hole systems the effect of the spacetime birefringence is an apparent time-dependent change in the inclination angle between the binary system's orbital angular momentum and the line-of-sight to the detector. (This change is apparent in the same sense that light is "bent" upon passage nearby a strongly gravitating object.) Sufficiently long observations of a binary system will enable this apparent rotation to be distinguished from the real rotation caused by spin-orbit and spin-spin angular momentum interactions in the binary system. Observations of just this kind will be possible using the LISA gravitational wave detector [13, 14, 15], which will be able to observe the inspiral of massive black hole binaries at redshifts approaching 30 for periods of a year or more. Gravitational wave observations of these systems with LISA may thus provide the first test of string theory or other quantum theories of gravity: yet another way in which gravitational wave observations can act a unique tool for probing the fundamental nature of the universe.

\section{Acknowledgments}

We are grateful to S. James Gates Jr. for encouraging us to look into the possibility of testing string theories with the Green-Schwarz term. We also thank Lubos Motl, Benjamin Owen, Michael Peskin, Andy Strominger, Emanuele Berti, Clifford Will, Carlos Sopuerta, Eric Poisson and Scott Hughes for enlightening discussions and comments.

SA acknowledges the support of the Eberly College of Science. NY acknowledges support from National Science Foundation awards PHY 05-55628 and PHY 02-45649. LSF acknowledges the support of NSF awards PHY 0653462 and PHY 05-55615, and NASA award NNG05GF71G. Lastly, we acknowledge the support of the Center for Gravitational Wave Physics, which is funded by the National Science Foundation under Cooperative Agreement PHY 01-14375.

[1] J. Polchinski, String theory. Volume 2: Superstring theory and beyond, vol. 2 (Cambridge University Press, Cambridge, UK, 1998).

[2] S. H. S. Alexander and J. Gates, S. James, JCAP 0606, 018 (2006), hep-th/0409014.

[3] A. Ashtekar, A. P. Balachandran, and S. Jo, Int. J. Mod. Phys. A4, 1493 (1989).

[4] L. Alvarez-Gaume and E. Witten, Nucl. Phys. B234, 269 (1984).

[5] S. Alexander and N. Yunes, A new ppn parameter to test chern-simons gravity (2007), hep-th/0703265.

[6] S. Alexander and N. Yunes, Parametrized post-newtonian expansion of chern-simons gravity (2007), arXiv:0704.0299 [hepth].

[7] T. L. Smith, A. L. Erickcek, R. R. Caldwell, and M. Kamionkowski (2007), arXiv:0708.0001 [astro-ph].

[8] R. Jackiw and S.-Y. Pi, Phys. Rev. D 68, 104012 (2003).

[9] S. H. S. Alexander, Is cosmic parity violation responsible for the anomalies in the WMAP data? (2006), hep-th/0601034.

[10] A. Lue, L.-M. Wang, and M. Kamionkowski, Phys. Rev. Lett. 83, 1506 (1999), astro-ph/9812088.

[11] M. Li, J.-Q. Xia, H. Li, and X. Zhang, Phys. Lett. B B651, 357 (2007), hep-ph/0611192.

[12] S. Alexander and J. Martin, Phys. Rev. D 71, 063526 (2005), hep-th/0410230.

[13] S. Merkowitz and J. C. Livas, eds., Laser Interferometer Space Antenna: Sixth International LISA Symposium, no. 873 in American Institute of Physics Conference Proceedings, Goddard Space Flight Center (American Institute of Physics, Melville, New York, 2007), proceedings of the Sixth International LISA Symposium.

[14] J. Baker, P. bender, P. Binetruy, J. Centrella, T. Creighton, J. Crowder, C. Cutler, K. Danzman, S. Drasco, L. S. Finn, et al., Tech. Rep. LISA-LIST-RP-436, Jet Propulsion Laboratory (2007), available at http://www.lisa-science.org/resources/talks-articles/science.

[15] Committee on NASA's Beyond Einstein Program: An Architecture for Implementation and Space Studies Board, Board on Physics and Astronomy, and Division on Engineering and Physical Sciences of the National Research Council, NASA's Beyond Einstein Program: An Architecture for Implementation (National Academies Press, Washington, DC, 2007).

[16] C. M. Will, Living Reviews in Relativity 9 (2006).

[17] D. M. Eardley, D. L. Lee, A. P. Lightman, R. V. Wagoner, and C. M. Will, Phys. Rev. Lett. 30, 884 (1973).

[18] D. M. Eardley, D. L. Lee, and A. P. Lightman, Phys. Rev. D 8, 3308 (1973).

[19] L. S. Finn, Class. Quantum Grav. 2, 381 (1985).

[20] C. Cutler and L. Lindblom, Phys. Rev. D 54, 1287 (1996), gr-qc/9601047. 
[21] F. D. Ryan, Phys. Rev. D 56, 1845 (1997).

[22] C. M. Will, Phys. Rev. D 57, 2061 (1998), gr-qc/9709011.

[23] L. S. Finn and P. J. Sutton, Phys. Rev. D 65, 044022 (2002).

[24] P. J. Sutton and L. S. Finn, Class. Quantum Grav. 19, 1355 (2002), gr-qc/0112018.

[25] C. M. Will and N. Yunes, Class. Quantum Grav. 21, 4367 (2004), ISSN 0264-9381, gr-qc/0403100.

[26] E. Berti, A. Buonanno, and C. M. Will, Class. Quantum Grav. 22, S943 (2005), ISSN 0264-9381, gr-qc/0504017.

[27] E. Berti, A. Buonanno, and C. M. Will, Phys. Rev. D 71, 084025 (2005), ISSN 0556-2821, gr-qc/0411129.

[28] P. D. Scharre and C. M. Will, Phys. Rev. D 65, 042002 (2002), gr-qc/0109044.

[29] O. Dreyer et al., Class. Quantum Grav. 21, 787 (2004), gr-qc/0309007.

[30] E. Berti, V. Cardoso, and C. M. Will, Phys. Rev. D 73, 064030 (2006), ISSN 1550-7998, gr-qc/0512160.

[31] C. W. Misner, K. S. Thorne, and J. A. Wheeler, Gravitation (W. H. Freeman \& Co., San Francisco, 1973).

[32] S. J. Waldman and The LIGO Scientific Collaboration (The LIGO Scientific Collaboration), Class. Quantum Grav. 23, S653 (2006), URL http://stacks .iop.org/0264--9381/23/S653

[33] F. Acernese, P. Amico, M. Alshourbagy, F. Antonucci, S. Aoudia, S. Avino, D. Babusci, G. Ballardin, F. Barone, L. Barsotti, et al., Class. Quantum Grav. 23, S635 (2006), URL http://stacks.iop.org/0264--9381/23/S635

[34] B. Willke, P. Ajith, B. Allen, P. Aufmuth, C. Aulbert, S. Babak, R. Balasubramanian, B. W. Barr, S. Berukoff, A. Bunkowski, et al., Class. Quantum Grav. 23, 207 (2006).

[35] H. Lück, M. Hewitson, P. Ajith, B. Allen, P. Aufmuth, C. Aulbert, S. Babak, R. Balasubramanian, B. W. Barr, S. Berukoff, et al., Class. Quantum Grav. 23, 71 (2006).

[36] P. Bender, P. Binetruy, S. Buchman, J. Centrella, M. Cerdonio, N. Cornish, M. Cruise, C. Cutler, K. Danzmann, L. S. Finn, et al., Tech. Rep. LISA-ScRD-004, LISA Project Office, NASA GSFC, Greenbelt, MD 20771 (2007).

[37] J. Baker, P. Bender, P. Binetruy, J. Centrella, T. Creighton, J. Crowder, C. Cutler, K. Danzman, S. Drasco, L. S. Finn, et al., Tech. Rep. LISA-LIST-RP-436, LISA Project Office, LISA Mission Science Office, Jet Propulsion Laboratory (2007).

[38] A. Vecchio, Phys. Rev. D 70, 042001 (2004), ISSN 0556-2821.

[39] L. S. Finn, Phys. Rev. D 46, 5236 (1992).

[40] L. S. Finn and D. F. Chernoff, Phys. Rev. D 47, 2198 (1993).

[41] S. H. Alexander, M. E. Peskin, and M. M. Sheikh-Jabbari, Gravi-leptogenesis: Leptogenesis from gravity waves in pseudoscalar driven inflation models (2007), hep-ph/0701139.

[42] R. Dijkgraaf, A. Sinkovics, and M. Temurhan, Adv. Theor. Math. Phys. 7, 1155 (2004), hep-th/0211241.

[43] P. Svrcek and E. Witten, JHEP 06, 051 (2006), hep-th/0605206.

[44] C.-Y. Sun and D.-H. Zhang (2006), hep-th/0611101.

[45] D. H. Wesley, P. J. Steinhardt, and N. Turok, Phys. Rev. D 72, 063513 (2005), hep-th/0502108.

[46] S. Alexander, R. H. Brandenberger, and D. Easson, Phys. Rev. D 62, 103509 (2000), hep-th/0005212.

[47] T. Battefeld and S. Watson, Rev. Mod. Phys. 78, 435 (2006), hep-th/0510022.

[48] R. H. Brandenberger, A. Nayeri, S. P. Patil, and C. Vafa (2006), hep-th/0608121.

[49] R. Brandenberger (2007), hep-th/0702001.

[50] P. Brax, C. van de Bruck, and A.-C. Davis, Rept. Prog. Phys. 67, 2183 (2004), hep-th/0404011.

[51] R. H. Brandenberger and C. Vafa, Nucl. Phys. B316, 391 (1989).

[52] A. A. Tseytlin and C. Vafa, Nucl. Phys. B372, 443 (1992), hep-th/9109048.

[53] R. Brandenberger, D. A. Easson, and D. Kimberly, Nucl. Phys. B623, 421 (2002), hep-th/0109165.

[54] A. Nayeri, R. H. Brandenberger, and C. Vafa, Phys. Rev. Lett. 97, 021302 (2006), hep-th/0511140.

[55] A. Garcia, F. W. Hehl, C. Heinicke, and A. Macias, Class. Quantum Grav. 21, 1099 (2004), gr-qc/0309008.

[56] K. G. Arun, B. R. Iyer, B. S. Sathyaprakash, S. Sinha, and C. van den Broeck, Phys. Rev. D 76, 104016 (2007), arXiv:0707.3920.

[57] C. Van Den Broeck and A. S. Sengupta, Binary black hole spectroscopy (2006), gr-qc/0610126.

[58] In the case of string theory, the absence of a Chern-Simons term leads to the Green-Schwarz anomaly. Quantum consistency requires cancellation of this anomaly. In order to eliminate the anomaly, the introduction of a Chern-Simons term is essential. Heterotic M-theory makes use of an anomaly inflow, which also leads to the same requirement.

[59] In [8], $C_{a b}$ was defined as a 4-dimensional generalization of the 3-dimensional Cotton tensor. However, such a generalization already exists [55] and differs from that introduced in [8]. Therefore, we shall here refer to this quantity as the C-tensor.

[60] One can show that a transverse-traceless gauge exists in Chern-Simons gravity, following the same steps as in classical general relativity [31]

[61] This parameterization, while convenient for counting, is not the most appropriate for describing a coalescing binary, where spins, eccentricity, and orbital plane orientation all evolve with time.

[62] See [56] for an analysis that relaxes this approximation

[63] This applies also to the assumption of zero eccentricity: if, as is likely, the estimation errors associated with eccentricity and $\xi$ are correlated it will be necessary to include an eccentricity-like parameter in the analysis in order to avoid underestimating the errors associated with the measurement of $\xi$.

[64] This approximation is weakest for the spin and orbital angular momentum parameters: see [57].

[65] Other theories, such as loop quantum gravity, may also possess a similar correction in the low-energy limit; however, no effective Hamiltonian has yet been found in the full theory of loop quantum gravity and cosmology, which prevents further speculation as to whether such an extension of general relativity requires a Chern-Simons modification. 